\title{
Opposing actions of histone deacetylase 1 and Notch signalling restrict expression of erm and fgf20a to hindbrain rhombomere centres during zebrafish neurogenesis
}

\author{
ELEWYS G. LIGHTMAN, MICHAEL R.M. HARRISON and VINCENT T. CUNLIFFE* \\ MRC Centre for Developmental and Biomedical Genetics, Department of Biomedical Science, \\ University of Sheffield, Sheffield, U.K.
}

\begin{abstract}
The rate and pattern of neurogenesis in the developing vertebrate nervous system are controlled by a complex interplay of intercellular signalling pathways and transcriptional control mechanisms. In the zebrafish hindbrain, Fgf20a promotes transcription of the gene encoding the ETS-domain transcription factor Erm in the non-neurogenic centres of rhombomeres. Here, we demonstrate that the epigenetic regulator, Histone Deacetylase 1 (Hdac1) and the Notch signalling pathway have opposing functions in regulating expression of both erm and fgf20a in the zebrafish hindbrain. Our results show that Hdac1 is required for expression of erm and fgf20a in rhombomeres, and that the Hdac1-dependent expression of these two genes is attenuated in rhombomere boundary regions by Notch signalling activity, thereby restricting erm and fgf20a transcripts to narrow stripes of cells at rhombomere centres.
\end{abstract}

KEY WORDS: neurogenesis, transcription, Hdac1, Notch, zebrafish

\section{Introduction}

Throughout the developing vertebrate central nervous system (CNS), proliferating multipotent neural progenitors give rise to a remarkable variety of neuronal and glial cell types. Some of the crucial steps underlying lineage restriction and the commitment of neural progenitors to specific differentiated neuronal or myelinating glial fates include progenitor withdrawal from the cell cycle, transcriptional silencing of neural progenitor determinants and transcriptional activation of genes encoding bHLH transcription factors such as ascl1b, neurog 1 (Bertrand et al., 2002) and olig2 (Rowitch, 2004). However, the processes driving commitment of proliferating progenitors to neuronal and glial fates in the CNS are countered by the regulated expression of neural progenitor maintenance factors such as the SoxB1 proteins (Pevny and Placzek, 2005) as well as the regulated activity of the Wnt, Notch and FGF signalling pathways (Ille and Sommer, 2005; Louvi and ArtavanisTsakonas, 2006; Gonzalez-Quevedo et al., 2010), which provide negative feedback that limits the rate and pattern of neurogenesis.

Histone deactylases (HDACs) are components of the epigenetic machinery that regulates gene transcription during embryonic development (Cunliffe, 2008). In zebrafish and the mouse, the class I HDAC, Hdac1, plays important roles in the transformation of neural progenitors into neurones and myelinating glia during development of the vertebrate CNS, by promoting cell cycle exit and transcription of neurogenic genes (Cunliffe, 2004; Yamaguchi et al., 2005; Stadler et al., 2005; Cunliffe and Casaccia-Bonnefil, 2006; Harrison et al., 2011; Montgomery et al., 2009; Ye et al., 2009). In the hindbrain, hdac1 is required for the specification of branchiomotor neurones and oligodendrocytes (Cunliffe, 2004; Cunliffe and Casaccia-Bonnefil, 2006). Our previous studies demonstrated that Hdac1 functions in the hindbrain by a mechanism that involves promoting expression of transcription factors such as those encoded by proneural genes, attenuating Notch target gene expression, and enabling neural fate-determining responses to Hedgehog pathway activity (Cunliffe, 2004; Cunliffe and CasacciaBonnefil, 2006; Harrison et al., 2011).

Accumulating evidence suggests that FGF-regulated transcription factors, such as the ETS-domain proteins Erm, Pea3 and Etv5, play important roles in neural specification and/or patterning within the developing CNS (Gonzalez-Quevedo et al., 2010; Raible and Brand, 2001; Roussigné and Blader, 2006). In the hindbrain, transcripts encoding Erm and its positive regulator Fgf20a are restricted

Abbreviations used in this paper: ETS, E26 Transformation-Specific; Erm, ETS-related molecule; fgf, fibroblast growth factor; hdacl, histone deacetylase 1.

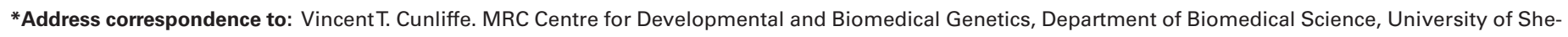
ffield, Western Bank, Sheffield S10 2TN, U.K..Tel: +44-114-222-2389. Fax: +44-114-222-2787. e-mail: v.t.cunliffe@sheffield.ac.uk 
to stripes of tissue within rhombomeres (Roussigné and Blader, 2006; Gonzalez-Quevedo et al., 2010), and recent work suggests that Erm could function in the establishment of Fgf20a-dependent, non-neurogenic zones at rhombomere centres (Gonzalez-Quevedo et al., 2010). Here, we demonstrate opposing roles for Hdac1 and Notch pathway signalling activity in regulating expression of both erm and fgf20a in the zebrafish hindbrain, which further suggests crucial functions for this transcription factor and its upstream signal in regulation of neurogenesis.

\section{Results}

\section{Hdac1 promotes expression of the ETS transcription factor} gene erm in the developing embryonic CNS

Previous studies in this laboratory revealed that in the zebrafish hindbrain, loss of hdac1 function severely impairs neuronal and glial differentiation without affecting the primary patterning of the neuroepithelium, including its subdivision into rhombomeres (Cunliffe, 2004). We wondered whether Hdac1 might therefore regulate expression of genes involved in neuronal patterning within rhombomeres, such as the transcription factor gene erm, fgf20adependent transcription of which is restricted to a series of robust stripes that are located at rhombomere centres (Roussigné and Blader, 2006; Gonzalez-Quevedo et al., 2010). We addressed this question by first analysing expression of ermin wild-type and hdac1 mutant embryos at 21, 24 and 31 hours post-fertilisation (hpf), which correspond to developmental time points shortly before and after the morphological phenotype of hdac1 mutant embryos first becomes apparent (Cunliffe, 2004). At $21 \mathrm{hpf}$, erm is expressed in the hindbrain of wild-type sibling embryos in a series of clearly demarcated stripes at rhombomere centres, with the strongest expression corresponding to stripes of tissue that are located within rhombomeres 4, 5 and 6. erm expression is also abundant within other regions of the $21 \mathrm{hpf}$ embryo, including the midbrainhindbrain boundary, posterior spinal cord, somites, tailbud, midbrain, optic vesicles and forebrain (Fig. 1). In 21 hpf hdac1 mutant embryos, erm expression in the hindbrain is limited to a weaker, more diffuse expression domain encompassing rhombomeres 4, 5 and 6 . Reduced expression of erm was also observed in the midbrain and midbrain-hindbrain boundary of the hdac1 mutant CNS, whereas erm transcript levels in the trunk and tailbud are relatively unaffected. By $24 \mathrm{hpf}$, a strong, centrally-located stripe of erm expression is visible in each of rhombomeres 2-7 (Fig. 1).

Fig. 1. Hdac1 is required to establish and maintain stripes of erm expression within the centres of hindbrain rhombomeres 2-7. In situ hybridisation analysis of erm expression in $(\mathbf{A}, \mathbf{C}, \mathbf{E}, \mathbf{G}, \mathbf{I}, \mathbf{K})$ wild-type sibling and (B,D,F,H,J,L) homozygous hdac1 mutant embryos, at 21 hpf (A-D), 24 hpf (E-J) and 31 hpf $(\mathbf{K}, \mathbf{L})$. Lateral views show significant reduction of erm expression in the hindbrain of hdac1 mutant embryos (B,F) compared to wild-type siblings $(A, E)$. Dorsal views of hindbrain are shown in panels $C, D, G, H$. Transverse sections through rhombomere 5 are shown in panels $I, J, K, L$. The hindbrain of wild-type sibling embryos $(C, G, I, K)$ exhibits robust stripes of erm expression at 21 and $24 \mathrm{hpf}$ that are restricted to rhombomere centres $(C, G)$. Both the ventricular zone and mantle region of the hindbrain express erm at $24 \mathrm{hpf}$, but by $31 \mathrm{hpf}$ erm expression is restricted to the ventricular zone. In hdac1 mutant embryos erm is expressed at $21 \mathrm{hpf}$ in a weak, more diffuse domain that encompasses rhombomeres 4, 5 and 6. By 24hpf, erm expression is extinguished in all rhombomeres of the hdac1 mutant hindbrain apart from rhombomere 4.
By contrast, in the hindbrain of 24 hpf hdac1 mutant embryos, expression of erm is extinguished in all rhombomeres apart from rhombomere 4 (Fig. 1), where expression persists, albeit relatively weakly. Transverse sections of the wild-type hindbrain in $24 \mathrm{hpf}$ embryos at the level of rhombomere 5 reveal a broad domain of erm expression encompassing both the ventricular zone and
A
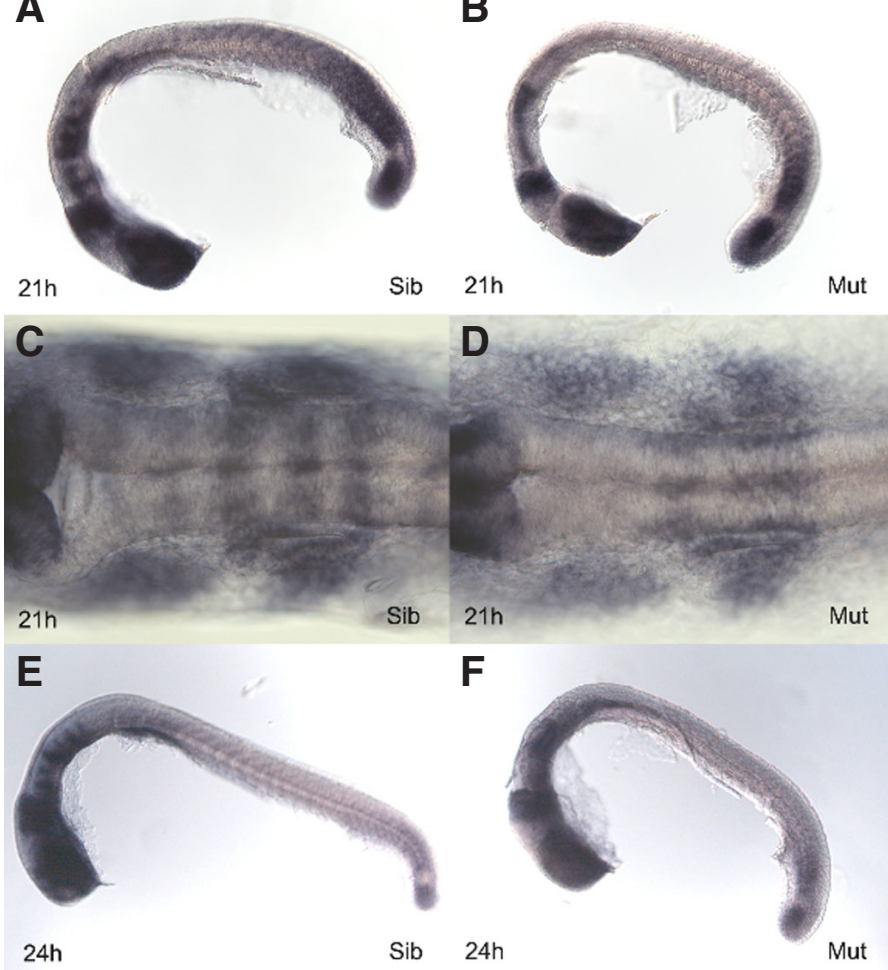

B

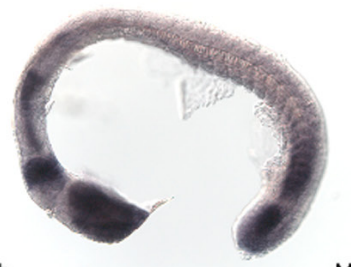

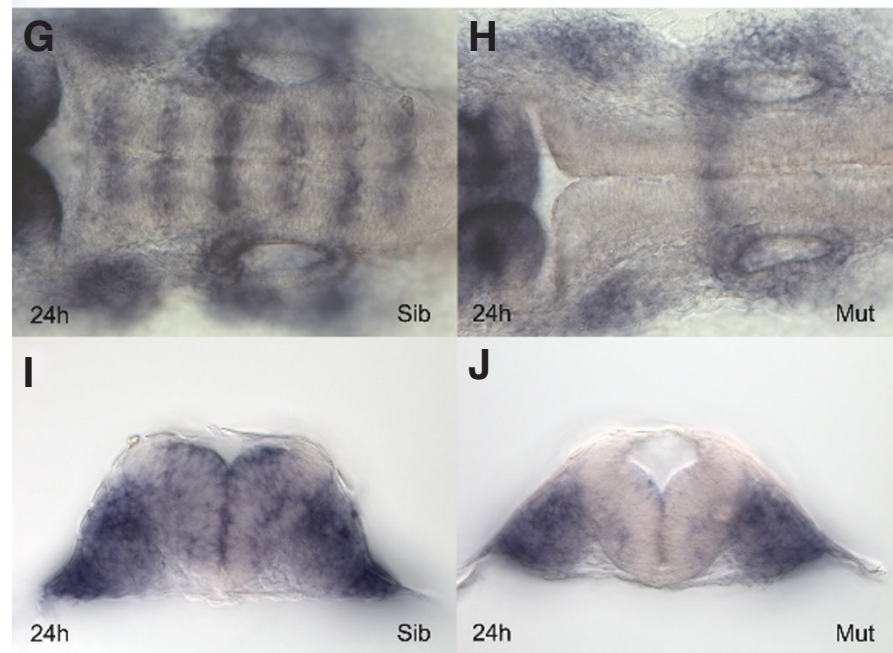

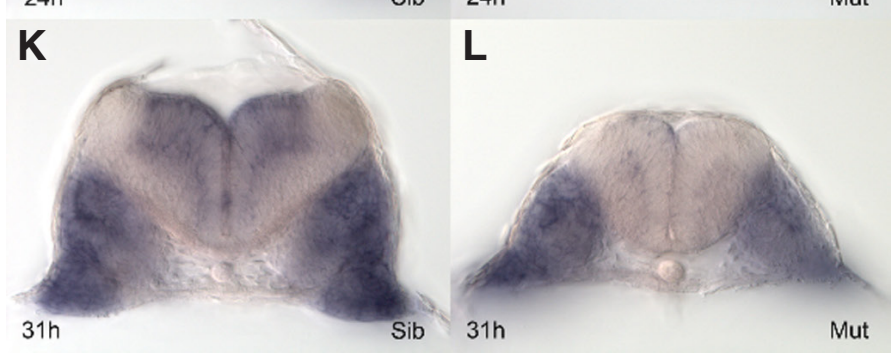


mantle region, which includes all but the lateral-most margins of the hindbrain. At $31 \mathrm{hpf}$, each stripe of erm expression is restricted to a T-shaped domain which encompasses the majority of the ventricular zone, but which excludes its lateral margins as well as the underlying mantle region (Fig. 1). In contrast, transverse sections through rhombomere 5 of hdac1 mutant embryos reveal a near complete loss of erm expression in this rhombomere at both $24 \mathrm{hpf}$ and $31 \mathrm{hpf}$. Taken together, these results demonstrate a key role for Hdac1 to maintain erm transcription in an expression domain that initially encompasses both the ventricular zone and the mantle region in hindbrain rhombomere centres, but which then becomes restricted to the ventricular zones at rhombomere centres. In addition, we conclude that expression of erm in rhombomere 4 is less sensitive to loss of hdac1 function than is the case for other rhombomeres, because a weak stripe of erm transcription persists

\section{A}

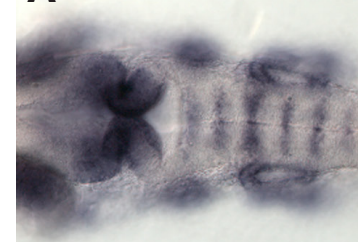

$24 h$

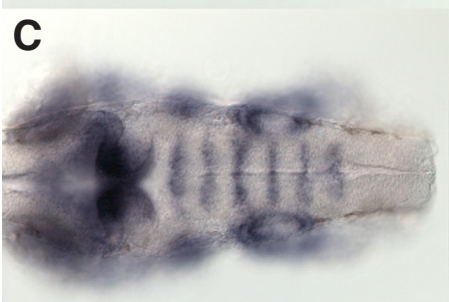

$27 \mathrm{~h}$

E

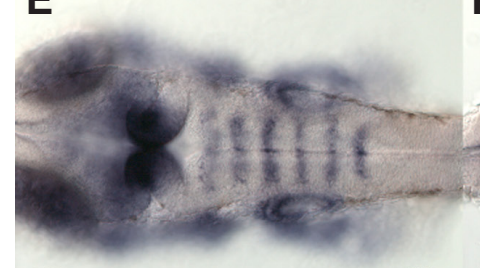

$30 \mathrm{~h}$

G

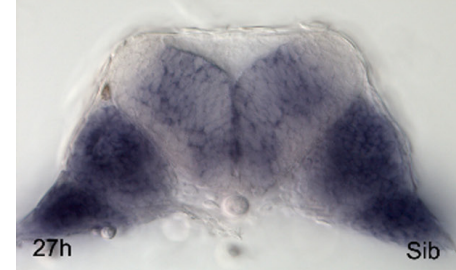

I

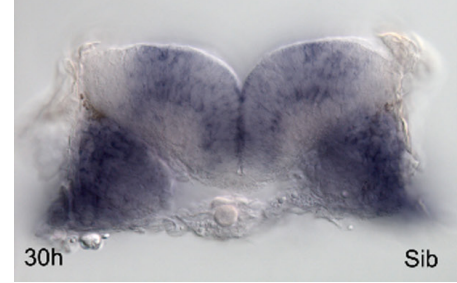

B

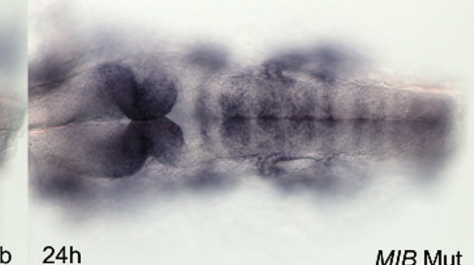

D

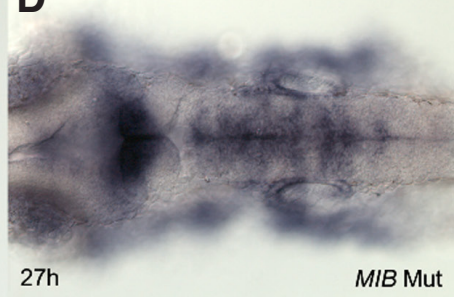

$\mathbf{F}$

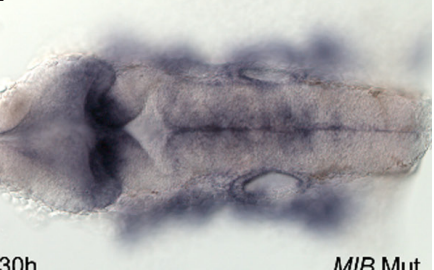

H

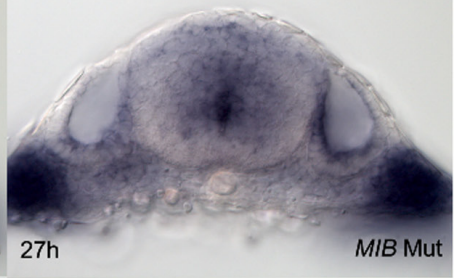

J

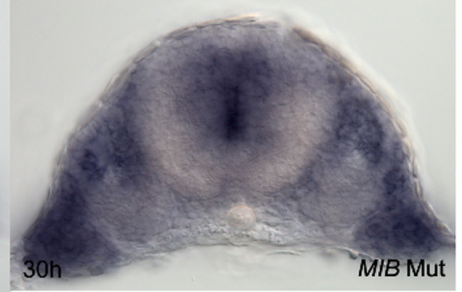

in hdac1 mutants when other domains of erm transcription in the hindbrain are extinguished.

\section{The Notch signalling pathway inhibits expression of the ETS transcription factor geneerm in the developing embryonic CNS}

Our previous studies demonstrated that Hdac1 promotes the transformation of proliferating progenitors into neurones and oligodendrocytes by activating a programme of neurogenic gene expression that includes proneural and other genes encoding transcription factors (Cunliffe, 2004; Cunliffe and Casaccia-Bonnefil, 2006; Harrison et al., 2011). Moreover, Hdac1 attenuates the expression of the Notch pathway target gene her6 and opposes the neural progenitor maintenance function of Notch signalling during CNS development. To determine whether Notch signalling regulates erm expression in the hindbrain ventricular zone, the expression pattern of erm was compared in the hindbrains of wildtype embryos and homozygous mind bomb mutants, which lack Notch pathway activity (Itoh et al., 2003; Bingham et al., 2003). This in situ hybridisation analysis revealed that mind bomb mutant embryos exhibit an expanded domain of ermexpression in hindbrain rhombomeres 2-7 both anteriorly and posteriorly, towards each rhombomere boundary, at 24, 27 and $30 \mathrm{hpf}$ (Fig. 2). In transverse sections through the hindbrain at the level of rhombomere 5 , the expression domain of erm in mind bomb mutant embryos is centrally located within the hindbrain and excluded from a superficial ring of tissue at the ventral and lateral limits of the hindbrain. By contrast, wild-type sibling embryos exhibited narrow stripes of erm expression that were restricted to the ventricular zone at rhombomere centres. Thus, we conclude that in wild-type embryos, Notch signalling represses erm expression in the boundary regions of each rhombomere.

\section{erm expression in the hindbrain ventricular zone is strictly dependent on Hdac1 function and limited by Notch pathway activity}

The opposing activities of Hdac1 and Notch signalling in the regulation of neuronal specification have previously been demonstrated (Cunliffe, 2004). We therefore carried out an epistatic analysis of the relationship between the requirements for hdac1 function and Notch signalling in the regulation of erm expression (Fig. 3). erm expression was analysed in $27 \mathrm{hpf}$ mind bomb mutants and wild-type siblings after microinjection with either an hdac1-specific translation-blocking morpholino (Hdac1ATG1) or

Fig. 2. Restriction of erm expression to narrow stripes at the centres of rhombomeres 2-7 is dependent on Notch signaling. In situ hybridisation analysis of erm expression in (A, $\mathbf{C}, \mathbf{E}, \mathbf{G}, \mathbf{I})$ wild-type sibling and $(\mathbf{B}, \mathbf{D}, \mathbf{F}, \mathbf{H}, \mathbf{J})$ homozygous mind bomb mutant embryos, at $24 \mathrm{hpf}(\mathbf{A}, \mathbf{B}), 27 \mathrm{hpf}(\mathbf{C}, \mathbf{D}, \mathbf{G}, \mathbf{H})$ and $30 \mathrm{hpf}(\mathbf{E}, \mathbf{F}, \mathbf{l}, \mathbf{J})$. Dorsal views of hindbrain are shown in panels A-F. Transverse sections through rhombomere 5 are shown in panels G-J. The hindbrain of wild-type sibling embryos $(A, C, E)$ exhibits narrow stripes of erm expression at 24, 27 and $30 \mathrm{hpf}$ that are restricted to rhombomere centres. However, in 24, 27 and 30 hpf mind bomb mutant embryos, erm is expressed in much broader domains within rhombomeres $(B, D, F)$, forming a near-continuous domain of erm expression that extends across rhombomeres 2-7. In transverse sections through the hindbrain of 27 and $30 \mathrm{hpf}$ mind bomb mutant embryos $(\mathrm{H}, \mathrm{J})$, a recognisable ventricular zone cannot be distinguished and erm expression is restricted to a central territory within hindbrain tissue, being most intense at the midline. By contrast, in age-matched wild-type sibling embryos $(G, I)$, erm expression is restricted to the clearly recognisable ventricular zone. 

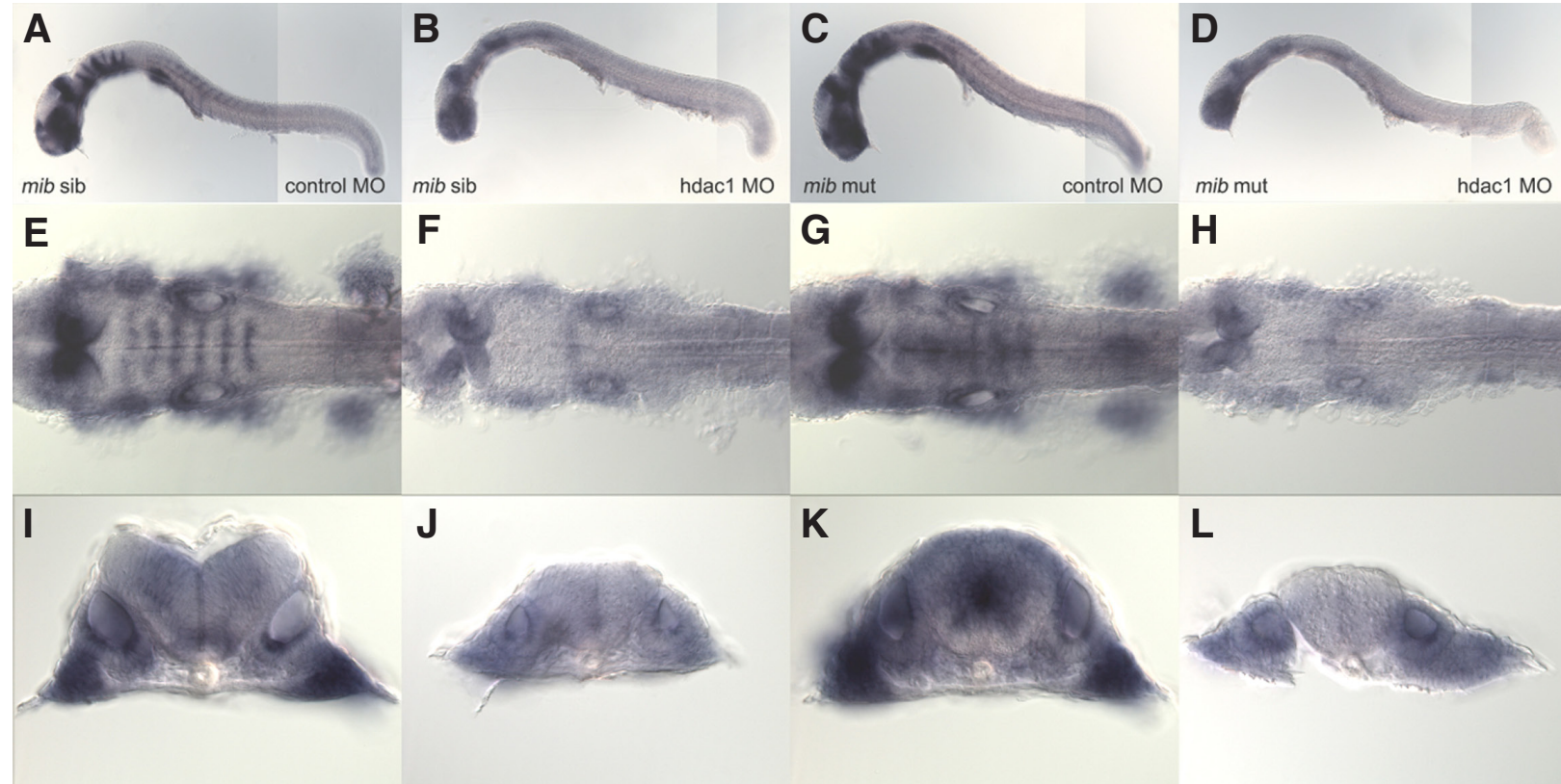

F

G
J

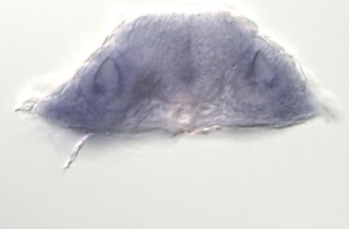

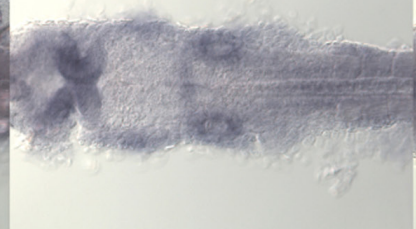

K

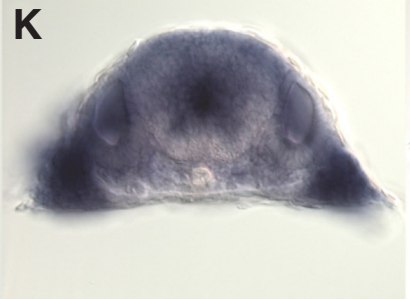

H

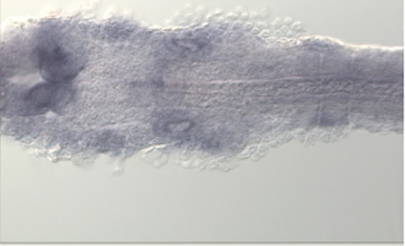

L

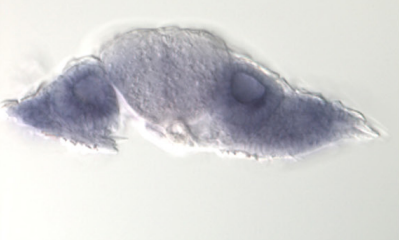

Fig. 3. Hdac1 acts epistatically and in opposition to the inhibitory effect of Notch signalling on erm transcription in the hindbrain ventricular zone. In situ hybridisation analysis of erm expression in $(\mathbf{A}, \mathbf{E}, \mathbf{I})$ wild-type sibling embyos injected with the hdac1 mismatch control morpholino, (B,F,J) wild-type sibling embryos injected with the hdac1ATG1 morpholino, (C, $\mathbf{G}, \mathbf{K})$ mind bomb mutant embryos injected with the hdac1 mismatch control morpholino, and (D, H, L) mind bomb mutant embryos injected with the hdac1ATG1 morpholino. Panels show lateral views (A-D), dorsal views of hindbrain (E-H), and transverse sections through rhombomere 5 (I-L) of 27 hpf embryos. Expression of erm is almost completely extinguished in the hindbrain of both hdac1ATG1 morphant embryos $(B, F, J)$ and mind bomb mutants microinjected with the hdac1ATG1 morpholino $(D, H, L)$, whereas the erm expression domain is expanded in the hindbrain of mind bomb mutants $(C, G, K)$, and remains restricted to stripes at rhombomere centres in wild-type siblings microinjected with the hdac1 mismatch control morpholino $(A, E, I)$.

an hdac1 mismatch control morpholino (Cunliffe, 2004). Wild-type sibling embryos that were microinjected with the hdac1 mismatch control morpholino displayed a wild-type erm expression pattern in the hindbrain that consists of a series of distinctive stripes within the rhombomere centres. Transverse sections through rhombomere 5 revealed that these stripes of expression were confined to the hindbrain ventricular zone. Wild-type sibling embryos that were microinjected with the Hdac1ATG1 morpholino exhibited a phenotype similar to that of hdac1 mutant embryos, in which the stripes of erm expression in rhombomeres 2-7 were completely extinguished, apart from the persistence of a weak stripe of erm transcription in rhombomere 4. Consistent with these observations, transverse sections through rhombomere 5 showed a complete absence of erm expression. By contrast, homozygous mind bomb mutant embryos microinjected with the hdac1 mismatch control morpholino displayed a phenotype that was identical to that observed in homozygous mind bomb mutants, where the

Fig. 4. Expression of fgf20a in the hindbrain is restricted to rhombomere centres by the opposing actions of Hdac1 and Notch signalling. In situ hybridisation analysis of fgf20a expression in $(\mathbf{A}, \mathbf{C})$ wild-type sibling, (B) hdac1 mutant and (D) mind bomb mutant embryos at 27hpf (A-D). Dorsal views of hindbrain are shown in each panel. Wild-type sibling embryos $(A, C)$ exhibit weak, narrow stripes of fgf20a expression in the hindbrain at $27 \mathrm{hpf}$ that are localised to rhombomere centres (signals in rhombomeres 4, 5 and 6 are indicated by red arrowheads). fgf20a expression is extinguished in the hindbrain of hdac1 mutant embryos (B). By contrast, mind bomb mutant embryos exhibit increased and ectopic expression of fgf20a in a broad domain within the hindbrain that encompasses multiple adjacent rhombomeres (D). erm expression domain within each rhombomere is expanded to fill the entire anterior-posterior extent of the rhombomere, leading to widespread and continuous expression of erm throughout the hindbrain (Fig. 3). Intriguingly, mib mutant embryos microinjected with the Hdac1ATG1 morpholino developed a phenotype in which ermexpression was almost completely extinguished within the hindbrain. This phenotype was indistinguishable from that of wild-type embryos injected with the Hdac1ATG1 morpholino and that of hdac1 mutant embryos, which indicated that the hindbrain phenotype of hdac1 morphant embryos is epistatic to that of homozygous mib mutants. Thus, the derepression of erm that occurs on either side
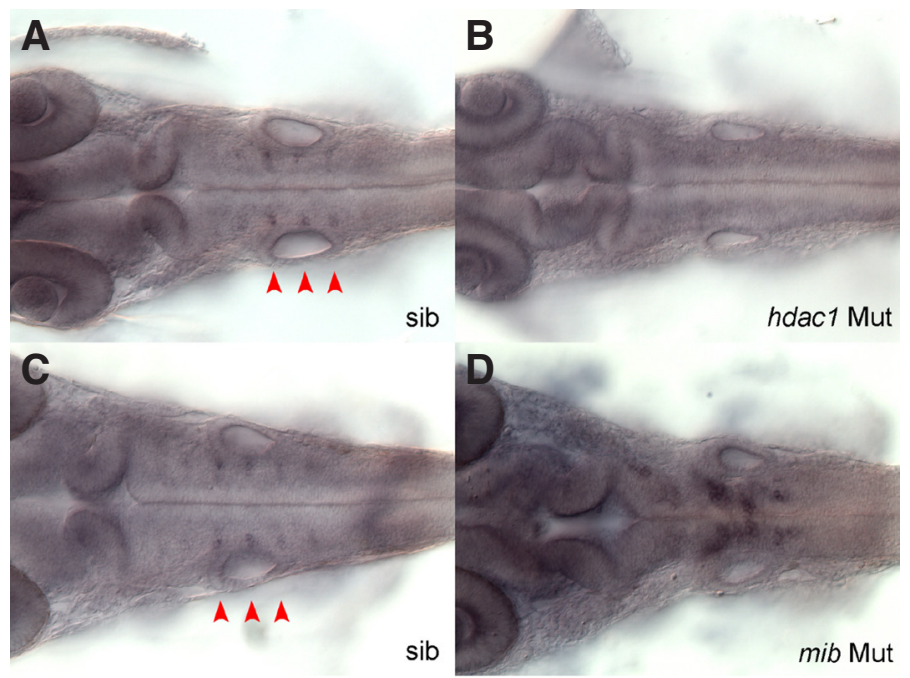
of rhombomere centres in mind bomb mutant embryos, like the restricted expression of erm at rhombomere centres in wild-type embryos, is strictly dependent on hdac1 function.

Taken together, our results indicate that expression of ermin the hindbrain ventricular zone is facilitated by Hdac1 and inhibited by Notch signalling. Moreover, the results of our epistasis experiments imply that Notch signalling attenuates Hdac1-driven transcription of erm in the ventricular zone specifically at rhombomere boundaries, thereby restricting erm expression to a narrow stripe of ventricular zone tissue at each rhombomere centre.

\section{Expression of fgf20a in the hindbrain requires Hdac1 function and is restricted to rhombomere centres by Notch signalling}

Expression of erm in the hindbrain ventricular zone is strictly dependent on fgf20a function in a subset of neurones within the underlying mantle region (Gonzalez-Quevedo et al., 2010). We therefore sought to determine whether, as for erm, the expression pattern of fgf20a in the hindbrain is influenced by Hdac1 function and Notch signalling. Fig. 4 shows that fgf20a expression is extinguished in the hindbrain of hdac1 mutant embryos. By contrast, the hindbrains of mind bomb mutant embryos exhibit increased and ectopic expression of fgf20a, giving rise to an extensive domain of strong fgf20a expression that extends across rhombomeres 4, 5 and 6. Taken together with our analysis of erm expression in hdac1 and mind bomb mutant embryos, these observations suggest that the gene expression changes observed for erm in hdac1 mutant and mind bomb mutant embryos are likely to be consequences of the effects of these mutations on transcription of fgf20a in mantle region neurones.

\section{Discussion}

Previous studies of the function of Hdac1 in development of the zebrafish nervous system have revealed important roles for this epigenetic regulator in facilitating the transformation of neural progenitors into neurones and oligodendrocytes. Hdac1 promotes transcription of a core programme of neurogenic regulators, including proneural proteins and other bHLH transcription factors (Cunliffe, 2004; Cunliffe and Casaccia-Bonnefil, 2006; Harrison et al., 2011), and it also attenuates transcription of neural progenitor marker genes, such as the Notch target her6 (Cunliffe, 2004; Harrison et al., 2011). In the zebrafish hindbrain, Hdac1 functions by rendering neural progenitors competent to respond to the Hedgehog signalling pathway, thereby directing their differentiation into postmitotic neurones (Cunliffe, 2004). Intriguingly, whilst hindbrain neurogenesis and neuronal patterning is severely defective in hdac1 mutant embryos, the primary patterning processes that establish rhombomeres and define their identities are relatively unaffected by loss of hdac1 function (Cunliffe, 2004). The spatial control of neurogenesis within rhombomeres is regulated by mechanisms involving Notch-mediated inhibition of neuronal differentiation at rhombomere boundaries (Cheng et al., 2004), and by FGF signalling from fgf20a-expressing neurons in the hindbrain mantle region (Gonzalez-Quevedo et al., 2010), which inhibits neurogenesis in the overlying tissue at rhombomere centres. Thus, the domains within which neuronal specification occurs in the hindbrain are defined by the activities of two distinct inhibitory signalling pathways. Here we show that expression of the FGF-regulated gene erm in the nonneurogenic central region of each rhombomere, and expression of fgf20a in the underlying mantle region, are strictly dependent on the function of Hdac1. These observations are consistent with the known role of Hdac1 as a key regulator of a core neurogenic programme in the zebrafish CNS, since the proneural gene expression that underlies neuronal differentiation in the mantle region, and expression of other markers of post-mitotic neurones, are almost completely extinguished in hdac1 mutant embryos (Cunliffe, 2004; Harrison et al., 2011). The restriction of erm expression to the ventricular zone at rhombomere centres implies a specific function for the Erm transcription factor in neural progenitors, which is consistent with other studies showing a close correlation between loss of erm expression and ectopic neurogenesis at rhombomere centres in homozygous fgf20amutant embryos (Gonzalez-Quevedo et al., 2010). Further studies will aim to explore the role of Erm in regulation of neural progenitor behaviour at rhombomere centres. Our results show that loss of Notch pathway activity expands the expression domains of both erm and fgf20a in the hindbrain. The expanded domain of erm transcription observed in the mind bomb mutant hindbrain is likely to be a consequence of the increased fgf20a expression that results from the ectopic and precocious induction of neuronal differentiation that occurs in mind bomb mutants. Thus, we infer that Notch signalling most likely inhibits erm transcription in the ventricular zone by inhibiting fgf20atranscription in the underlying mantle region at the anterior and posterior ends of each rhombomere. Such a possibility is also consistent with the additional observation that derepression of erm expression in the mind bomb mutant hindbrain is strictly dependent on neurogenesispromoting Hdac1 (Fig. 3), implying that Hdac1-dependent feedback from neurons in the mantle region, in the form of secreted Fgf20a protein, induces erm expression in the overlying ventricular zone. Future studies will aim to investigate the relationships between the Notch and FGF signalling pathways and the role of Hdac1 in mediating interactions between these two pathways in neural progenitors and their differentiated neuronal progeny.

\section{Materials and Methods}

\section{Zebrafish stocks}

hdac $1^{\text {hi1618 }}$ and mind bomb $b^{\text {ta52b }}$ mutant zebrafish were maintained at the University of Sheffield. Animal care and use was in accordance with the UK Animals (Scientific Procedures) Act 1986.

\section{Microinjection of morpholinos}

Morpholino sequences were as follows: Hdac1ATG1: 5'-ttg ttc ctt gag aac tca gcg cca t-3'; Hdac1 Mismatch control: 5'-ttg ctc gtt gag aac tct gca cca t-3'.

1-2 $\mathrm{nl}$ of $0.3 \mathrm{mM}$ morpholino solution in milli- $Q$ water was microinjected into embryos at the 1-2-cell stage. Embryos were maintained in E3 culture medium at $28.5^{\circ} \mathrm{C}$ until required for fixation overnight in $4 \%$ paraformaldehyde at $4^{\circ} \mathrm{C}$, then subsequently dehydrated in methanol and stored at $-20^{\circ} \mathrm{C}$ until required for in situ hybridisation.

\section{In situ hybridisation analysis of gene expression}

Digoxigenin-labelled RNA probes were prepared as recommended by the manufacturer (Roche). Whole-mount in situ hybridisation was performed using standard procedures (Oxtoby and Jowett, 1993). Details of the erm probe utilised are available on request.

\section{Acknowledgements}

This research was funded by a Wellcome Trust Project Grant to VTC (WT081884MA), an MRC postgraduate studentship to MRMH and a bur- 
sary from the MRC Centre for Developmental and Biomedical Genetics to EGL. Zebrafish facilities were supported through MRC Centre awards to Professor PW Ingham (Grants G0400100 and G0700091).

\section{References}

BERTRAND, N., CASTRO, D.S. and GUILLEMOT, F. (2002). Proneural genes and the specification of neural cell types. Nature Rev Neurosci 3: 517-530.

BINGHAM, S., CHAUDHARI, S., VANDERLAAN, G., ITOH, M., CHITNIS, A. and CHANDRASEKHAR,A. (2003). Neurogenic phenotype of mind bomb mutant leads to severe patterning defects in the zebrafish hindbrain. Dev Dyn 228: 451-463.

CHENG, Y.C., AMOYEL, M., QIU, X., JIANG, Y.J., XU, Q. and WILKINSON, D.G. (2004). Notch activation regulates the segregation and differentiation of rhombomere boundary cells in the zebrafish hindbrain. Dev Cell 6: 539-550.

CUNLIFFE, V.T. (2004). Histone deacetylase 1 is required to repress Notch target gene expression during zebrafish neurogenesis and to maintain the production of motoneurones in response to hedgehog signalling. Development 131:2983-2995.

CUNLIFFE, V.T. and CASACCIA-BONNEFIL, P. (2006). Histone deacetylase 1 is essential for oligodendrocyte specification in the zebrafish CNS. Mech Dev123:24-30.

CUNLIFFE, V.T. (2008). Eloquent silence: developmental functions of Class I Histone deacetylases. Curr Opin Genet Dev 18: 404-410.

GONZALEZ-QUEVEDO, R., LEE, Y., POSS, K.D. and WILKINSON, D.G. (2010). Neuronal regulation of the spatial patterning of neurogenesis. Dev Cell18:136-147.

HARRISON M.R.M., GEORGIOU, A.S., SPAINK H.P. and CUNLIFFE, V.T. (2011). The epigenetic regulator Histone Deacetylase 1 promotes transcription of a core neurogenic programme in zebrafish embryos. BMC Genomics 12:24.

ILLE, F. and SOMMER, L. (2005). Wnt signalling: multiple functions in neural development. Cell Mol Life Sci 62: 1100-1108.

ITOH, M., KIM, C.H., PALARDY, G., ODA, T., JIANG, Y.J., MAUST, D., YEO, S.Y., LORICK, K., WRIGHT, G.J., ARIZA-MCNAUGHTON, L., WEISSMANN, A.M.,
LEWIS, J., CHANDRASEKHARAPPA, S.C. and CHITNIS, A.B. (2003). Mind bomb is a ubiquitin ligase that is essential for efficient activation of Notch signaling by Delta. Dev Cell 4: 67-82.

LOUVI, A., and ARTAVANIS-TSAKONAS, S. (2006). Notch signalling in vertebrate neural development. Nature Rev Neurosci 7: 93-102.

MONTGOMERY, R.L., HSIEH, J., BARBOSA, A.C., RICHARDSON, J.A. and OLSON E.N. (2009). Histone deacetylases 1 and 2 control the progression of neural precursors to neurons during brain development. Proc NatIAcad SciUSA 106: 7876-7881.

OXTOBY, E. and JOWETT, T. (1993). Cloning of the zebrafish krox-20 gene ( $k r x-20)$ and its expression during hindbrain development. NuclAcids Res 21: 1087-1095.

PEVNY, L. and PLACZEK, M. (2005). SOX genes and neural progenitor identity. Curr Opin Neurobiol 15: 7-13.

RAIBLE, F. and BRAND, M. (2001). Tight transcriptional control of the ETS domain factors Erm and Pea3 by Fgf signalling during early zebrafish development. Mech Dev 107: 105-117.

ROUSSIGNE, M. and BLADER, P. (2006). Divergence in regulation of the PEA3 family of ETS transcription factors. Gene Exp. Patterns 6: 777-782.

ROWITCH, D.H. (2004). Glial specification in the vertebrate neural tube. Nature Rev Neurosci 5: 409-419.

STADLER, J.A., SHKUMATAVA, A., NORTON, W.H.J., RAU, M.J., GEISLER, R. FISCHER, S. and NEUMANN C.J. (2005). Histone deacetylase 1 is required for cell cycle exit and differentiation in the zebrafish retina. Dev Dyn 233: 883-889.

YAMAGUCHI, M., TONOU-FUJIMORI, N., KOMORI, A., MAEDA, R., NOJIMA, Y., LI, H.C., OKAMOTO, H. and MASAI, I. (2005). Histone deacetylase 1 regulates retinal neurogenesis in zebrafish by suppressing Wnt and Notch signaling pathways. Development 132: 3027-3043.

YE, F., CHEN, Y., HOANG, T., MONTGOMERY, R.L., ZHAO, X.H., BU, H., HU, T., TAKETO, M.M., VAN ES, J.H., CLEVERS, H., HSIEH, J., BASSEL-DUBY, R., OLSON, E.N. and LU, Q.R. (2009). HDAC1 and HDAC2 regulate oligodendrocyte differentiation by disrupting the $\beta$-catenin-TCF interaction. Nature Neurosci 12: 829-839. 


\section{Further Related Reading, published previously in the Int. J. Dev. Biol.}

Expression of protocadherin-19 in the nervous system of the embryonic zebrafish. Liu, Q., Chen, Y., Kubota, F., Pan, J.J. and Murakami, T. Int. J. Dev. Biol. (2010). 54: 905-911.

Competition for ligands between FGFR1 and FGFR4 regulates Xenopus neural development.

Yamagishi, $\mathrm{M}$. and Okamoto, $\mathrm{H}$.

Int. J. Dev. Biol. (2010). 54: 93-104.

Notch activity is required to maintain floorplate identity and to control neurogenesis in the chick hindbrain and spinal cord. Le Roux, I., Lewis J. and Ish-Horowicz, D.

Int. J. Dev. Biol. (2003). 47: 263-272.

Segmentation of the vertebrate hindbrain: a time-lapse analysis.

Kulesa, P. and Fraser, S.E.

Int. J. Dev. Biol. (1998). 42: 385-392.

Ets-1 and Ets-2 proto-oncogenes exhibit differential and restricted expression patterns during Xenopus laevis oogenesis and embryogenesis.

Meyer, D., Durliat, M., Senan, F., Wolff, M., Andre, M., Hourdry, J. and Remy, P.

Int. J. Dev. Biol. (1997). 41: 607-620

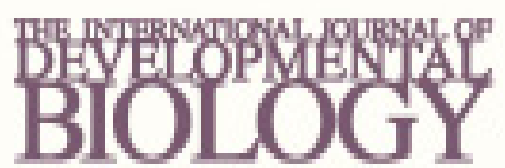

Houre st Har att
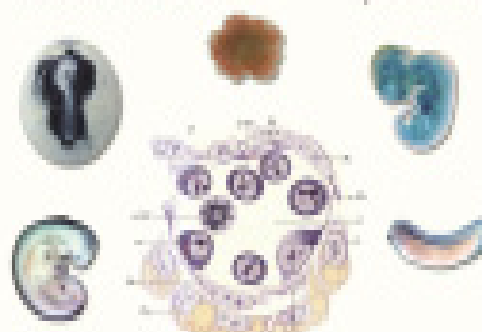

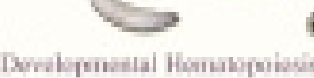

5 yr ISI Impact Factor $(2010)=2.961$

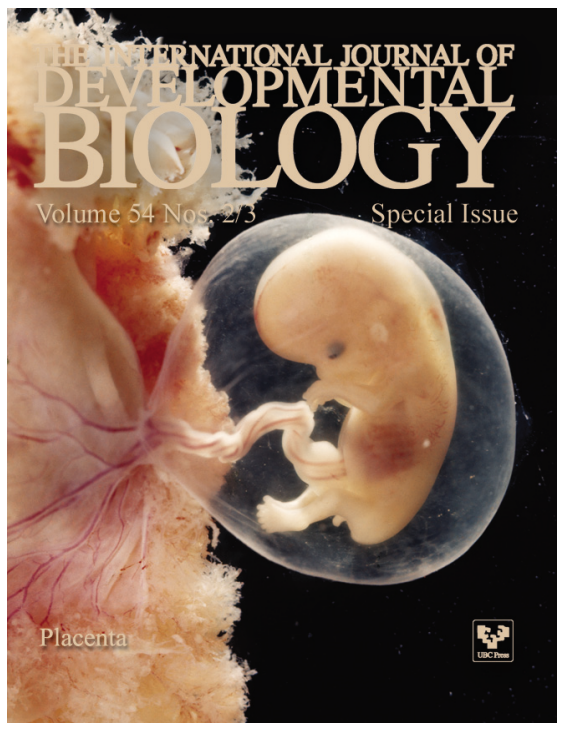

